\section{AN ANALYSIS OF THE EFFICIENCY OF METHODS OF TEMPERATURE ERROR CORRECTION IN SURFACE TEXTURE MEASUREMENTS} WLODZIMIERZ MAKIELA, JACEK SWIDERSKI, KRZYSZTOF STEPIEN

Kielce University of Technology, Faculty of Mechatronics and Mechanical Engineering, Department of Manufacturing Engineering and Metrology, Kielce, Poland DOI : 10.17973/MMSJ.2020_03_2019137

kstepien@tu.kielce.pl

One of the factors influencing contact measurements of surface texture are periodical changes of environmental temperature that are associated with the mode of operation of airconditioning systems applied in metrological laboratories. Temperature changes are the reason of appearing of additional waviness components in the measurement result. Research activities aiming at elimination of this type of error from measurement results have been conducted for a few years at the Department of Manufacturing Engineering and Metrology of the Kielce University of Technology. In order to separate the waviness resulting from periodical changes of environmental temperature various types of filters can be applied, including Gaussian filters, two-dimensional wavelet transform or Fast Fourier Transform algorithms. The paper presents up-to-date results of the analysis of the efficiency of individual filtration methods aiming at the correction of temperature error that appears when measuring surface texture with the use of contact methods.

KEYWORDS

Surface texture, contact measurement, stylus profilometer, temperature effect, profile filtering

\section{INTRODUCTION}

At present in industrial practice, characterization of the surface irregularities is conducted with the use of spatial analysis [Miller 2017, Zorawski 2016]. It is due to the fact that such method of analysis provides much more information about surface texture of the workpiece than an analysis based on a singular 2D profile [Stepien 2019, Gogolewski 2020]. Spatial measurements of surface texture can be carried out with the use of contact profilometry based on measurement data aquisition through the scanning along two mutually perpendicular directions [Hatala 2012].

One of the main drawbacks of contact profilometry is that it is a time-consuming method. Measurement time in this method depends on measurement area, speed of displacement of the measuring tip along the $\mathrm{X}$-axis, which is perpendicular to machining traces and sampling step along the $\mathrm{Y}$-axis (which corresponds to the number of profiles to be measured). Dimensions of measurement area depend on the character of irregularities occuring on the surface of the workpiece. Surfaces characterized by high value of roughness parameters require application of larger measurement area [Cep 2008]. Values of the speed of displacement of the measuring tip available in typical contact profilometers range between $0,1 \div 2 \mathrm{~mm} / \mathrm{s}$. Using the speed higher than $0,5 \mathrm{~mm} / \mathrm{s}$ is risky, as it can lead to appearance of a "flight" phenomenon. Flight phenomenon is the loss of the contact of the tip and the surface under investigation, which is caused by the dynamics of the tip relocation. Measurement of too low number of profiles along $\mathrm{Y}$-axis can, in some cases, lead to the loss of important information about specific features of measured surface.

\section{AIR-CONDITIONING TEMPERATURE EFFECT AS AN IMPORTANT FACTOR IN LONG-LASTING SURFACE TEXTURE MEASUREMENTS}

Requirements and limitations resulting from standards concerning surface texture measurements are the reason why the the measurement of surface stereometry can take from a few dozen of minutes to a few hours. In consequence, the significant factor affecting measurement results are the variations of environmental temperature, that under laboratory conditions are caused by the characteristics of an operation of an air-conditioning system periodical temperature change at amplitude dependent of the precision of applied airconditioning system. The influence of periodical temperature changes on results of measurements of surface stereometry with the use of profilometric method can be observed when measuring the AFL-type standard - a flat interference plate. The isometric view of such measurement in the direction of $Y$ axis reveals occurence of waviness component (see Fig. 1).

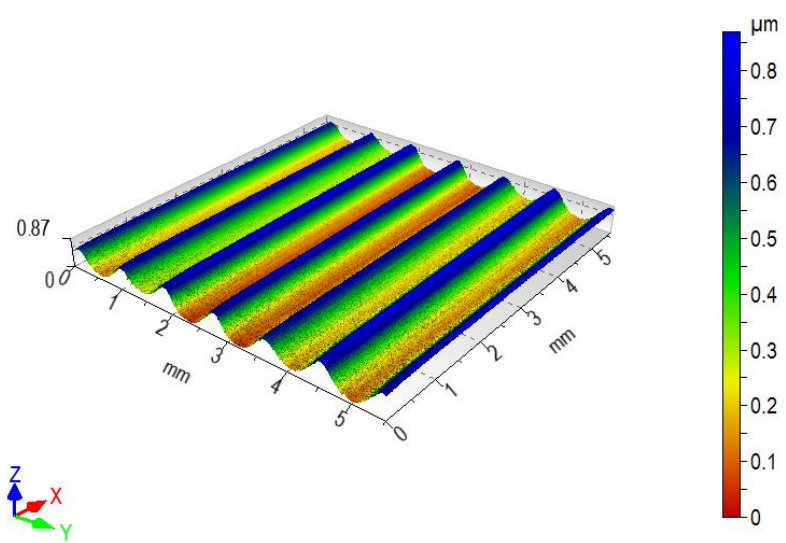

Figure 1. Isometric view of the measurement of the AFL standard

The wavines component is strictly correlated with the changes of environmental temperature (see Fig. 2).

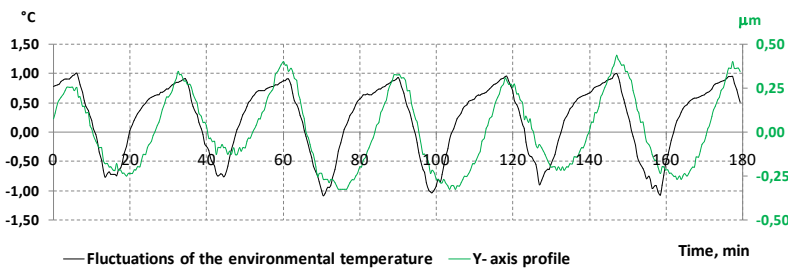

Figure 2. Comparison of changes environmental temperature and the changes of the values of the profile of AFL standard along Y-axis direction

The amplitude of changes of the profile values depends on the amplitude of the temperature changes during the measurement as well as on the design of the measuring instrument, in particular the quantitiy of measurement loop, consisting of such elements of the profilometer, as: the fundamental, the column, the traverse unit, the sensor, the workpiece and the measuring table allowing displacement of the workpiece along $\mathrm{Y}$-axis direction 


\section{OF A THERMIC COVER}

The waviness component along $\mathrm{Y}$-axis is a systematic error, whose influence can be compensated by an application of the cover that prevents the profilometer from affecting environmental conditions or through removal of this component from the signal with the use of various types of filters.

Usually profilometers characterized by low measurement uncertainty are equipped with a special-purpose cover that isolates the system from affecting environmental conditions. An example of such instrument is contact profilometer Form Talysurf PGI 1200 (see Fig. 3). Owing to the application of interferometric transducer, whose range is $12,5 \mathrm{~mm}$ and the resolution equal to $0,8 \mathrm{~nm}$, this instrument is able to conduct surface stereometry measurements concerning form, waviness and roughness. The instrument is equipped with a measuring table allowing displacement of the workpiece along $\mathrm{Y}$-axis with the step of $1 \mu \mathrm{m}$. The measuring tip of the instrument is made of diamond and it has shape of cone, whose vertex angle is ? $=90^{\circ}$ and the vertex radius is rtip $=2$ ? $\mathrm{m}$. Measurement speed ranges from $0,1 \mathrm{~mm} / \mathrm{s}$ do $2 \mathrm{~mm} / \mathrm{s}$.

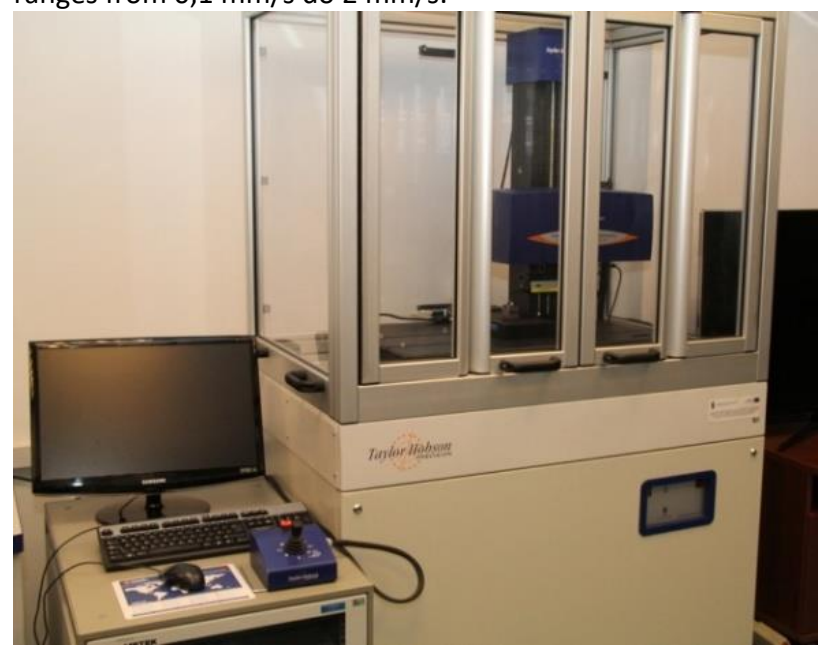

Figure 3. Contact profilometer Form Talysurf PGI 1200 equipped with a isolating cover

An efficiency of the cover has been investigated through the comparison of results of measurements of the temperature inside the cover and the temperature of the column of the profilometer when the air-conditioning system was turned on. Two cases were under investigation: when the cover was opened and when the cover was closed. Results of measurements are presented in Fig. 4.

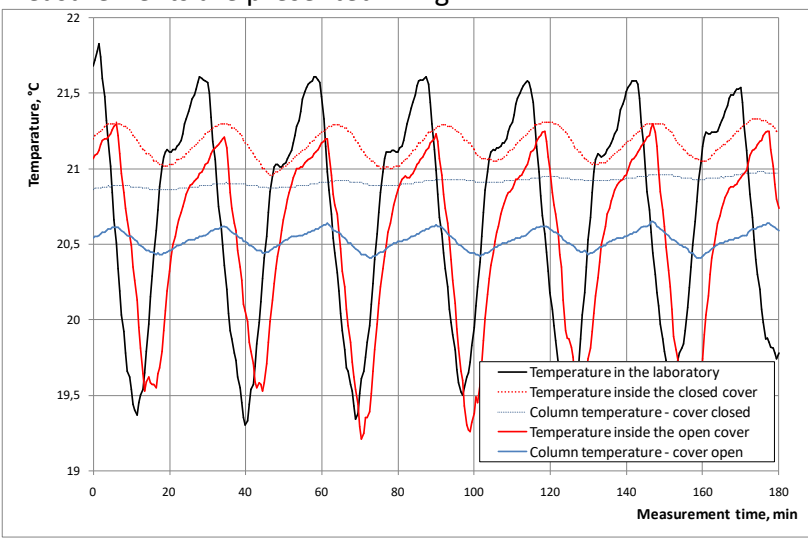

Figure 4. Results of temperature measurements

Average amplitude of temperature changes in the laboratory room was about $1.3^{\circ} \mathrm{C}$, the amplitude of temperature changes

inside closed cover was about $0.2^{\circ} \mathrm{C}$, whereas the amplitude of temperature changes of the column when the cover was closed was equal to about $0.06^{\circ} \mathrm{C}$.

The next stage of the experiment was to conduct measurements of AFL standard when the cover was opened and when it was closed. Measurements results are presented in Fig. 5.
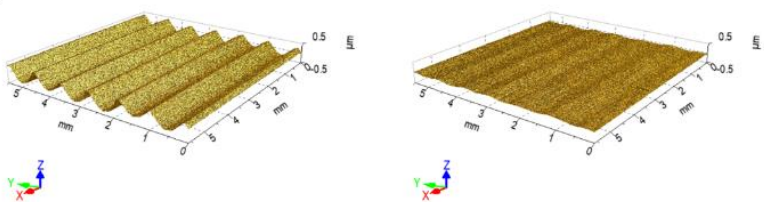

c)

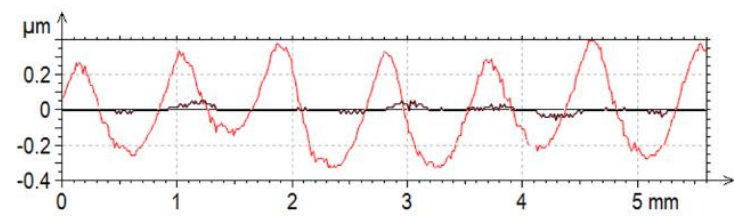

Figure 5. Results of AFL standard: a) an isometric view for the case of opened cover, b) an isometric view for the case of closed cover, c) comparison of selected 2D profiles of both surfaces in $\mathrm{Y}$-axis

An amplitude of waviness component of the surface along Yaxis resulting from periodical changes of environmental temperature when the cover was opened is much higher than it was for the case of closed cover. Thus, one can conclude that the application of the cover is quite efficient method to minimize the influence of changes of environmental temperature on measurement results.

Table 1 presents results of measurements of parameters of surface texture when the cover was opened and when the cover was closed. The results refer to unfiltered surfaces (surface $\mathrm{S}-\mathrm{F}$ ) containing components resulting from temperature changes.

\begin{tabular}{l|c|c|c|c|c|}
\multirow{2}{*}{ Cover } & \multicolumn{4}{|c}{ Surface texture parameters, $\mu \mathrm{m}$} \\
\cline { 2 - 6 } & $S a$ & $S q$ & $S p$ & $S v$ & $S z$ \\
\hline Open & 0,19 & 0,21 & 0,45 & 0,42 & 0,87 \\
\hline Closed & 0,02 & 0,03 & 0,11 & 0,16 & 0,27 \\
\hline
\end{tabular}

Table 1. Surface texture parameters for the case of opened and closed cover

Significant supressing of the amplitude of temperature changes for the case of closed cover results in obtaining much lower values of parameters describing the surface stereometry.

\section{COMPENSATION OF TEMPERATURE EFFECT WITH THE USE OF SIGNAL FILTERING}

Another method allowing compensation of an effect of changes of environmental temperature is to remove waviness components from the profile through filtering measurement data. In order to do that various types of filters can be used. In this work authors focus on applying of a few types of filters to compensate temperature error. The application of the filters was illustrated for ground surface. Figure 6 shows an isometric view of measured surface. The measurement was carried out with the use of contact profilometer without the cover, when the amplitude of periodical changes of environmental temperature in the laboratory room was about $0,5^{\circ} \mathrm{C}$. Measurement time was 360 minutes. Measurement results consist of 360 profiles. An analysis of the isometric view reveals 
that waviness component resulting from periodical changes of environmental temperature appears in the signal.

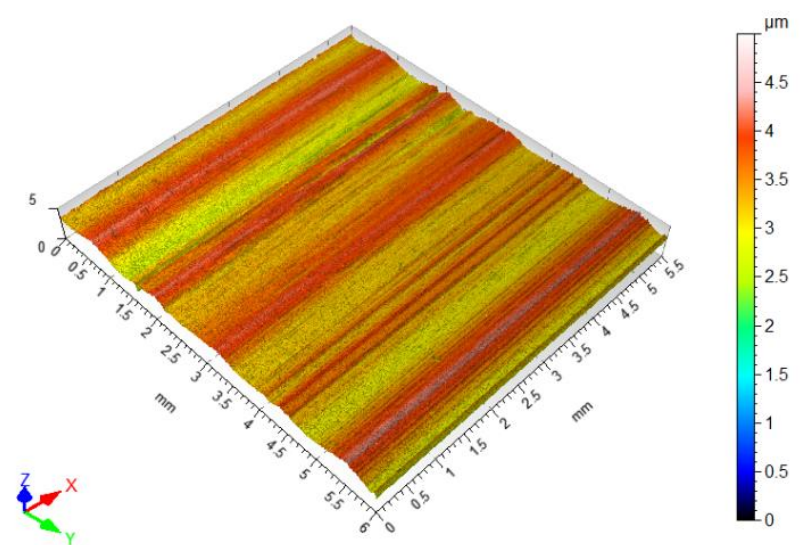

Figure 6. Isometric view of ground surface containing waviness resulting from the changes of environmental temperature

After selection of singular 2D profile and conducting harmonic analysis it is possible to determine the length of the wave resulting from periodical temperature changes. The harmonic analysis reveals that the wavelength $1,2 \mathrm{~mm}$ is the dominant one in the spectrum. Along $\mathrm{X}$-axis the profile is of random character. Analyzed 2D profile and the results of harmonic analysis are shown in Fig. 7 and in Fig. 8, respectively.

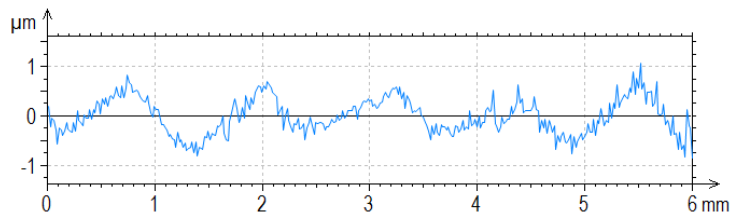

Figure 7. Analyzed 2D profile along Y-axis

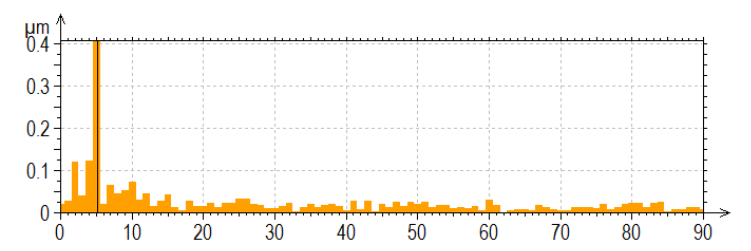

Wavelength $\# 5(1.20 \mathrm{~mm})$
Magnitude: $0.41 \mathrm{~mm}$

Figure 8. Harmonic analysis of preselected 2D profile along $Y$-axis

In order to compensate the temperature error following types of filter have been used: Gaussian filter, median filter, spline filter, Fourier filter and the wavelet filter (two-dimensional wavelet transform with Coiflet mother wavelet) [Janecki 2011, Janecki 2012]. In order to apply the filters it is fundamental to select correct parameters, permitting removal of waviness component resulting from the temperature error, only [Makieła 2019]. Figure 9 shows isometric views of measured surface after applying various types of filters.

Figure 10 presents comparison of preselected amplitude parameters of surface stereometry:

$\mathrm{Sa}$ - Arithmetic mean height

$\mathrm{Sq}$ - Root mean square height

$\mathrm{Sp}$ - Maximum peak height

Sv - Maximum pit height

$\mathrm{Sz}$ - Maximum height

for the primary surface containing the waviness component resulting from temperature error and for filtered surfaces. a)
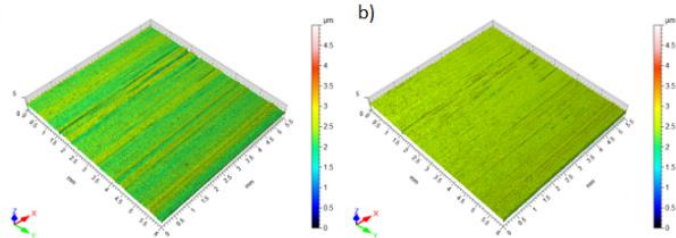

c)
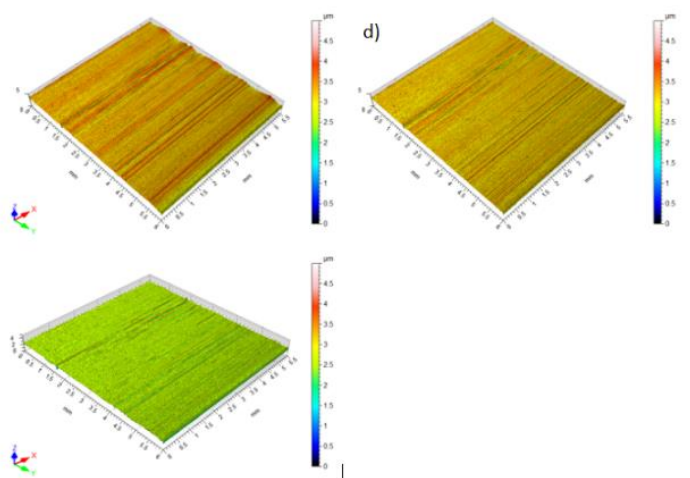

Figure 9. Isometric view of measured ground surface after applying of the filters: a) Gaussian filter, b) median filter, c) spline filter, d) Fourier filter, e) wavelet filter (2D wavelet transform with Coiflet mother wavelet)

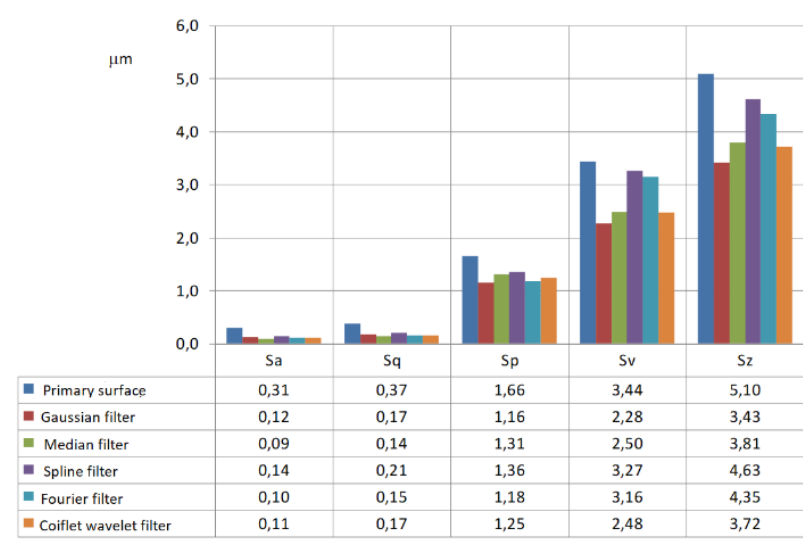

Figure 10. Values of amplitude parameters of surface stereometry for the primary surfaces and for filtered surfaces

Applied filtration methods are the reason of the fact that all amplitude parameters of filtered surfaces are lower than the ones relating to the primary surface. Figure 11 presents the character of the change caused by subsequent filter for $\mathrm{Sz}$ parameter. Continuous line refers to the $\mathrm{Sz}$ value of the primary surface, whereas dotted line refers to the mean value of $\mathrm{Sz}$ calculated for all applied filtration methods.

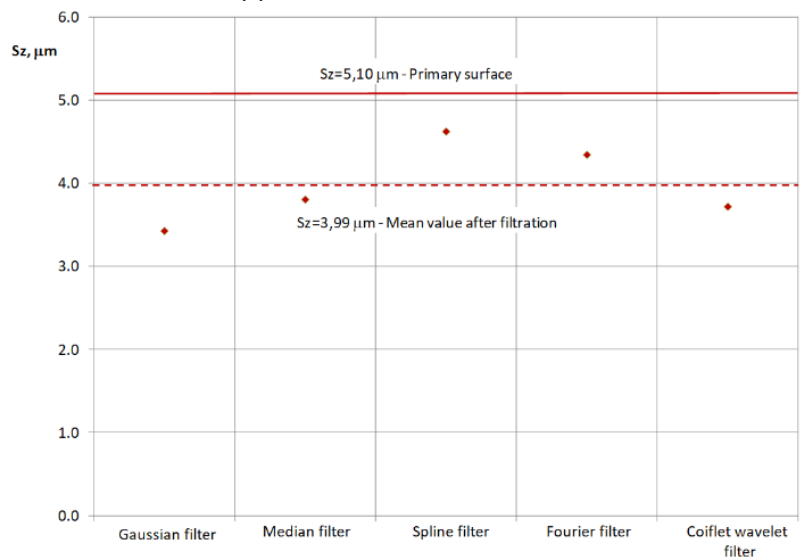

Figure 11. Values of Sz parameter for the primary surface and for filtered surfaces 
It is noticeable that the application of spline filter causes the smallest changes of the parameter. The most significant change of the parameter is the result of the application of Gaussian filter. For other types of filters, the character of the change of the parameter is very similar.

\section{CONCUSIONS}

Conducted experiments revealed significant influence of periodical changes of environmental temperature on results of surface texture for long-lasting measurements. The temperature changes contribute to the appearance of waviness component along Y-axis. An efficient method to minimize this effect is to apply thermic cover that significantly suppresses the amplitude of environmental temperature changes. In consequence, it brings about significant decrease of the waviness amplitude along Y-axis, which is, in fact., the systematic temperature error. If measuring system is not equipped with a cover, then an alternative method of minimization of temperature effect can be applied, which is based on application of suitable filtration method. Following types of filters can be applied to remove waviness components: Gaussian, median, spline, FFT filter or the filtration with the use of two-dimensional wavelet transform. Selection of suitable parameters of the filter or the basic wavelet requires a lot of experience from the operator, as there is a risk that apart from the waviness being an effect of the temperature error the filtration can remove real waviness components, too. It can contribute to decrease reliability of measurement results.

Conducted research addressed the problem of the surface for whose waviness component caused by the temperature error was dominant. This is the reason why further research work should focus on an evaluation of applicability of various types of filters for surfaces containing waviness components whose frequency is similar to the waviness being an effect of temperature changes.

\section{REFERENCES}

[Cep 2008] Cep, R., et al. Chip Formation Analysis During Hard Turning. Strojarstvo, 2008, Vol. 50, No. 6., pp 337-345. ISSN 0562-1887

[Gogolewski 2020] Gogolewski D., Influence of the edge effect on the wavelet analysis process. Measurement, 2020, Vol. 152, pp. 107314, doi.org/10.1016/j.measurement.2019.107314

[Hatala 2012] Hatala, M., et al. Research of the Technological Parameters Importance for Plasma Arc Thermal Cutting. Applied Mechanics and Materials, 2012, Vol. 110-116, pp 3742 3749. ISSN 1662-7482

[Janecki 2011] Janecki, D., Gaussian filters with profile extrapolation. Precision Engineering, 2011, Vol. 35, No. 4., pp 602-606. ISSN 0141-6359

[Janecki 2012] Janecki, D., Edge elimination effect elimination in the recursive implementation of Gaussian filters. Precision Engineering, 2012, Vol. 36, No. 1., pp 128-136. ISSN 0141-6359

[Makieła 2019] Makieła, W., et al. Identification of the minimum chip thickness based on the two-dimensional wavelet analysis. Journal of Physics: Conference Series, 2019, Vol. 1183, pp. 012008, doi:10.1088/1742-6596/1183/1/012008

[Miller 2017] Miller, T., et al. Influence of temperature gradient on surface texture measurements with the use of profilometry.
Bulletin of the Polish Academy of Sciences, February 2017, Vol.65, No.1., pp 53-61. ISSN 0239-7528

[Stepien 2019] Stepien K., et al., Images of the Machined Surface in Evaluation of the Efficiency of a Micro-Smoothing Process. Strojniški vestnik - Journal of Mechanical Engineering, 2019. Vol. 65, No. 7-8, pp 410-419.

[Zorawski 2016] Zorawski, W., et al. Mechanical properties and corrosion resistance of HVOF sprayed coatings using nanostructured carbide powders. Arch. Metall. Mater., 2016, Vol.61, No.4., pp 1839-1846. ISSN 1733-3490

\section{CONTACTS:}

Prof. Włodzimierz Makieła

Kielce University of Technology,

Department of Manufacturing Engineering and Metrology

Al. 1000-lecia P. P. 7, 25-314 Kielce, Poland

Phone: +48413424434

e-mail: wmakiela@tu.kielce.pl

MSc. Jacek Świderski

Kielce University of Technology,

Department of Manufacturing Engineering and Metrology

Al. 1000-lecia P. P. 7, 25-314 Kielce, Poland

Phone: +48413424342

e-mail: swiderski@tu.kielce.pl

Prof. Krzysztof Stępień

Kielce University of Technology,

Department of Manufacturing Engineering and Metrology

Al. 1000-lecia P. P. 7, 25-314 Kielce, Poland

Phone: + 48413424519

e-mail: kstepien@tu.kielce.pl 